\title{
Closed Form Continued Fraction Expansions of Special Quadratic Irrationals
}

\author{
Daniel Fishman ${ }^{1}$ and Steven J. Miller ${ }^{2}$ \\ ${ }^{1}$ Goldman, Sachs \& Co., 85 Broad Street, New York, NY 10004, USA \\ ${ }^{2}$ Department of Mathematics and Statistics, Williams College, Williamstown, MA 01267, USA
}

Correspondence should be addressed to Steven J. Miller; sjm1@williams.edu

Received 12 November 2012; Accepted 28 November 2012

Academic Editors: C. da Fonseca, D. S. Kim, and W. Menasco

Copyright (c) 2013 D. Fishman and S. J. Miller. This is an open access article distributed under the Creative Commons Attribution License, which permits unrestricted use, distribution, and reproduction in any medium, provided the original work is properly cited.

We derive closed form expressions for the continued fractions of powers of certain quadratic surds. Specifically, consider the recurrence relation $G_{n+1}=m G_{n}+\ell G_{n-1}$ with $G_{0}=0, G_{1}=1, m$ a positive integer, and $\ell= \pm 1$ (note that $m=\ell=1$ gives the Fibonacci numbers). Let $\varphi_{m, \ell}=\lim _{n \rightarrow \infty} G_{n} / G_{n-1}$. We find simple closed form continued fraction expansions for $\varphi_{m, \ell}^{k}$ for any integer $k$ by exploiting elementary properties of the recurrence relation and continued fractions.

\section{Introduction}

In [1], van der Poorten wrote that the elementary nature and simplicity of the theory of continued fractions is mostly well disguised in the literature. This makes one reluctant to quote sources when making a remark on the subject and seems to necessitate redeveloping the theory $a b$ initio. As our work is an outgrowth of [1], we happily refer the reader to that paper for some basic background information on continued fractions, and to the books [2, 3] for proofs. Briefly, every real number $\alpha$ has a continued fraction expansion

$$
\alpha=a_{0}+\frac{1}{a_{1}+\left(1 /\left(a_{2}+(1 / \cdot .)\right)\right)},
$$

where each $a_{i}$ is an integer (and a positive integer unless $i=0$ ). The $a_{i}$ 's are called the partial quotients. For brevity we often write

$$
\alpha=\left[a_{0}, a_{1}, a_{2}, \ldots\right]
$$

If we truncate the expansion at $a_{n}$, we obtain the $n$th partial quotient

$$
\frac{p_{n}}{q_{n}}=\left[a_{0}, a_{1}, a_{2}, \ldots, a_{n}\right]
$$

The $p_{n}$ 's and $q_{n}$ 's satisfy the very important relation

$$
p_{n} q_{n-1}-p_{n-1} q_{n}=(-1)^{n-1}
$$

Continued fractions encode much useful information about the algebraic structure of a number and frequently arise in approximation theory and dynamical systems. Clearly, $\alpha$ is rational if and only if its continued fraction is finite, and a beautiful theorem of Lagrange states that $\alpha$ is a quadratic irrational if and only if the continued fraction expansion is periodic.

In this paper, we explore the continued fraction expansions of powers of quadratic surds. Recall that a quadratic surd is an irrational number of the form $P \pm \sqrt{D} / Q$, where $P, Q \in \mathbb{Z}$ and $D$ is a nonsquare integer. By Lagrange's theorem we know these numbers and their powers have periodic continued fractions, which suggests many questions, such as what is the length of the period as well as what are the entries. Some special cases were done by van der Poorten in [1]. He studied solutions to Pell's equation $X^{2}-D Y^{2}=$ \pm 1 , ( $D$ a nonsquare integer). Using the solution, he derived expansions for the continued fraction of $\sqrt{D}$ and $(1+\sqrt{D}) / 2$ (with $D \equiv 1 \bmod 4$ ) and then for the expansions of some simple functions of these numbers as well as numbers related to Diophantine equations similar to Pell's equation. 
Another technique that shows promise in manipulating continued fractions comes from an unfinished paper of Gosper [4]. He develops a set of algorithms for finding closed form expressions of simple functions of a given quadratic irrational, as well as for more complicated functions combining quadratic irrationals. His paper addresses the need for finding a nice way of adding and multiplying the continued fractions of quadratic surds. The basic technique employed is an analog of Euclid's algorithm. This operation is carried out using two-dimensional arrays in the simple cases and then requires added dimensions when considering functions of two or more quadratic irrationals. Unfortunately, these algorithms, while useful for many applications, do not reveal the nature of the underlying structure in a closed form in an accessible manner.

The purpose of this paper is to continue these investigations for additional families. We derive closed form expressions for the continued fractions of powers of certain quadratic surds.

As the Fibonacci case is perhaps the most interesting, and some generalizations can be reduced to this case, we state the results there first. Let $f_{k}$ denote the $k$ th Fibonacci number and $F_{n}$ denote the $n$th Fibonacci number. The reason for using both upper and lower case letters for the Fibonacci numbers is to make the algebra in Section 2 visually easier to read by using upper case for the Fibonacci numbers whose subscript is variable. Set $\varphi=\lim _{n \rightarrow \infty} F_{n} / F_{n-1}$, which is the golden mean; note $\varphi^{k}=\lim _{n \rightarrow \infty} F_{n} / F_{n-k}$.

Theorem 1. One has the following:

$$
\frac{F_{n}}{F_{n-k}}= \begin{cases}{\left[f_{k+1}+f_{k-1}, \frac{F_{n-k}}{F_{n-2 k}}\right]} & \text { if } k \text { is even } \\ {\left[f_{k+1}+f_{k-1}-1,1, \frac{F_{n-k}}{F_{n-2 k}}-1\right]} & \text { if } k \text { is odd, }\end{cases}
$$

which yields

$$
\varphi^{k}= \begin{cases}{\left[\overline{f_{k+1}+f_{k-1}}\right]} & \text { if } k \text { is odd } \\ {\left[f_{k+1}+f_{k-1}-1, \overline{1, f_{k+1}+f_{k-1}-2}\right]} & \text { if } k \text { is even } .\end{cases}
$$

The techniques used to prove the above theorem can be extended to certain recurrence relations. Let $g_{k}=G_{k}$ for the same reason as before.

Theorem 2. Consider the recurrence relation $G_{n+1}=m G_{n}+$ $\ell G_{n-1}$ with $G_{0}=0, G_{1}=1, m$ a positive integer, and $\ell= \pm 1$, again letting $g_{k}=G_{k}$. Let

$$
\varphi_{m, \ell}=\lim _{n \rightarrow \infty} \frac{G_{n}}{G_{n-1}}=\frac{m \pm \sqrt{m^{2}+4 \ell}}{2} .
$$

Then, for any positive integer $k$, if $\ell=1$ one has

$$
\varphi_{m, 1}^{k}= \begin{cases}{\left[\overline{g_{k+1}+g_{k-1}}\right]} & \text { if } k \text { is odd } \\ {\left[g_{k+1}+g_{k-1}-1, \overline{1, g_{k+1}+g_{k-1}-2}\right]} & \text { if } k \text { is even },\end{cases}
$$

while if $\ell=-1$ one has

$$
\varphi_{m,-1}^{k}=\left[g_{k+1}-g_{k-1}-1, \overline{1,\left(g_{k-1}-g_{k-1}-2\right)}\right] .
$$

The numerical data that led us to these results, as well as some additional experimental observations which, as of now, we have not been able to isolate into general theorems, are available at [5].

On a personal note, this paper is an outgrowth of two undergraduate research classes taken by the first named author under the instruction of the second named author (and others) at Princeton University in 2002 and 2003. One of the topics covered was continued fractions, where many of Alf van der Poorten's papers [1, 6-15] were read and enjoyed. These types of questions are ideally suited to introduce students to research. The results here, while later discovered to have been proved through other techniques (see e.g., [16]) were originally found through numerical exploration, which suggested the proof strategy.

The second named author had the fortune of being at Brown University with Alf a few years later and remembers fondly numerous conversations on continued fractions and other topics in general, and the expansions in this paper in particular. Alf's constant enthusiasm, knowledge of the field, and helpful comments greatly improved the exposition of continued fractions in [3], a textbook developed from the Princeton course.

\section{Continued Fractions of Powers of the Golden Mean}

As we will see later that the proof of Theorem 2 reduces to the special case of the Fibonacci numbers, we study this case first. For notational convenience, we write $F_{n}$ for the $n$th Fibonacci number when $n$ varies and write $f_{k}$ for the $k$ th Fibonacci number when $k$ is a fixed input. This notation helps us visually parse the equations that follow.

The following lemma collects some well-known properties of the Fibonacci numbers which will be useful below. The proofs are standard (see e.g., [17], especially Chapter 5).

Lemma 3. The Fibonacci numbers satisfy the following properties.

(1) $F_{n}=F_{n-1}+F_{n-2}, F_{0}=0, F_{1}=1$.

(2) $\varphi=\lim _{n \rightarrow \infty}\left(F_{n} / F_{n-1}\right)=(1+\sqrt{5}) / 2$.

(3) Binet's Formula:

$$
F_{n}=\frac{1}{\sqrt{5}}\left[\left(\frac{1+\sqrt{5}}{2}\right)^{n}-\left(\frac{1+\sqrt{5}}{2}\right)^{n}\right] .
$$

(4) $F_{n-2} F_{n}-\left(F_{n-1}\right)^{2}=(-1)^{n-1}$.

(5) $F_{n}=f_{k+1} F_{n-k}+f_{k} F_{n-k-1}$. 
The relations discussed below were initially found by numerically exploring the finite continued fraction expansions of $F_{n} / F_{n-k}$. Note that for the golden mean $\varphi=$ $\lim _{n \rightarrow \infty} F_{n} / F_{n-1}$, we have

$$
\begin{gathered}
\varphi=\frac{1+\sqrt{5}}{2}=[1,1,1,1,1, \ldots], \\
\lim _{n \rightarrow \infty} \frac{F_{n}}{F_{n-k}}=\lim _{n \rightarrow \infty} \frac{F_{n}}{F_{n-1}} \frac{F_{n-1}}{F_{n-2}} \cdots \frac{F_{n-(k-1)}}{F_{n-k}}=\varphi^{k} .
\end{gathered}
$$

For $k=2$, it is trivial to find the continued fraction of $\varphi^{2}$ since $F_{n}=F_{n-1}+F_{n-2}$ implies

$$
\frac{F_{n}}{F_{n-2}}=1+\frac{F_{n-1}}{F_{n-2}}
$$

Taking the limit as $n \rightarrow \infty$, we find

$$
\varphi^{2}=\lim _{n \rightarrow \infty} \frac{F_{n}}{F_{n-2}}=1+\varphi=[2,1,1,1,1, \ldots] .
$$

With $k=3$, however, the result is not as obvious. From Lemma 3 (5) we note that $F_{n}=f_{3} F_{n-2}+f_{2} F_{n-3}=2 F_{n-2}+$ $F_{n-3}$, which implies

$$
\frac{F_{n}}{F_{n-3}}=1+2 \frac{F_{n-2}}{F_{n-3}}
$$

Unfortunately, in general there is no simple expression for the continued fraction of $2 \alpha$ given the expansion of $\alpha$. Numerical computations of the continued fraction expansions of $F_{n} / F_{n-3}$ were found to have the form $[4,4, \ldots, \rho]$, where $\rho$ is 3 if $n \equiv 1 \bmod 3,5$ if $n \equiv 2 \bmod 3$, and 4 if $n \equiv 0 \bmod 3$. This is easily proved, as after some algebra we find that

$$
\frac{F_{n}}{F_{n-3}}=4+\frac{1}{F_{n-3} / F_{n-6}},
$$

and the claim now follows from knowing the first few ratios. Further, we get the continued fraction expansion for $\varphi^{3}$ simply by taking limits.

Theorem 1 states that this algebra can be generalized to any $k$, as we now show.
Proof of Theorem 1. We have

$$
\begin{aligned}
\frac{F_{n}}{F_{n-k}}= & \frac{f_{k+1} F_{n-k}+f_{k} F_{n-k-1}}{F_{n-k}} \\
= & f_{k+1}+f_{k} \frac{F_{n-k-1}}{F_{n-k}} \\
= & f_{k+1}+\frac{f_{k-1} F_{n-k-1}+f_{k-2} F_{n-k-1}}{F_{n-k}} \\
= & f_{k+1}+\left(\left(f_{k-1} F_{n-k-1}+f_{k-1} F_{n-k-2}\right)\right. \\
& \left.\quad+f_{k-2} F_{n-k-1}-f_{k-1} F_{n-k-2}\right) \times\left(F_{n-k}\right)^{-1} \\
= & f_{k+1}+\frac{f_{k-1} F_{n-k}}{F_{n-k}}+\frac{f_{k-2} F_{n-k-1}-f_{k-1} F_{n-k-2}}{F_{n-k}} \\
= & \left(f_{k+1}+f_{k-1}\right)+\frac{f_{k-2} F_{n-k-1}-f_{k-1} F_{n-k-2}}{F_{n-k}} .
\end{aligned}
$$

As $F_{n-k-1}=f_{k} F_{n-k-k}+f_{k-1} F_{n-k-k-1}$ and $F_{n-k-2}=$ $f_{k-1} F_{n-k-k-1}+f_{k-2} F_{n-k-k-2}$, (16) becomes

$$
\begin{aligned}
\frac{F_{n}}{F_{n-k}}= & \left(f_{k+1}+f_{k-1}\right) \\
+ & \left(f_{k-2}\left(f_{k} F_{n-2 k}+f_{k-1} F_{n-2 k-1}\right)\right. \\
& \left.\quad-f_{k-1}\left(f_{k-1} F_{n-2 k}+f_{k-2} F_{n-2 k-1}\right)\right) \times\left(F_{n-k}\right)^{-1} \\
= & \left(f_{k+1}+f_{k-1}\right)+\frac{f_{k-2}\left(f_{k} F_{n-2 k}\right)-f_{k-1}\left(f_{k-1} F_{n-2 k}\right)}{F_{n-k}} \\
= & \left(f_{k+1}+f_{k-1}\right)+\frac{F_{n-2 k}\left[f_{k-2} f_{k}-\left(f_{k-1}\right)^{2}\right]}{F_{n-k}} .
\end{aligned}
$$

From Lemma 3 (4) we know that $f_{k-2} f_{k}-\left(f_{k-1}\right)^{2}=$ $(-1)^{k-1}$. Using this and (17), (16) becomes

$$
\begin{aligned}
\frac{F_{n}}{F_{n-k}} & =\left(f_{k+1}+f_{k-1}\right)+\frac{F_{n-2 k}(-1)^{k-1}}{F_{n-k}} \\
& =\left(f_{k+1}+f_{k-1}\right)+\frac{(-1)^{k-1}}{F_{n-k} / F_{n-2 k}} .
\end{aligned}
$$

If $k$ is odd then $(-1)^{k-1}=1$ and so (18) becomes

$$
\frac{F_{n}}{F_{n-k}}=\left(f_{k+1}+f_{k-1}\right)+\frac{1}{F_{n-k} / F_{n-2 k}},
$$

while if $k$ is even then $(-1)^{k-1}=-1$ and so (18) becomes

$$
\frac{F_{n}}{F_{n-k}}=\left(f_{k+1}+f_{k-1}\right)+\frac{-1}{F_{n-k} / F_{n-2 k}} .
$$

If $k$ is even we must manipulate further, and (20) becomes

$$
\frac{F_{n}}{F_{n-k}}=\left(f_{k+1}+f_{k-1}-1\right)+1-\frac{1}{F_{n-k} / F_{n-2 k}} .
$$


Now,

$$
\begin{aligned}
1-\frac{1}{F_{n-k} / F_{n-2 k}} & =\frac{F_{n-k}-F_{n-2 k}}{F_{n-k}} \\
& =\frac{1}{F_{n-k} /\left(F_{n-k}-F_{n-2 k}\right)} \\
& =\frac{1}{1+\left(F_{n-2 k} /\left(F_{n-k}-F_{n-2 k}\right)\right)} \\
& =\frac{1}{1+\left(1 /\left(\left(F_{n-k}-F_{n-2 k}\right) / F_{n-2 k}\right)\right)} \\
& =\frac{1}{1+\left(1 /\left(\left(F_{n-k} / F_{n-2 k}\right)-1\right)\right)} .
\end{aligned}
$$

Taking the limit as $n \rightarrow \infty$ yields

$$
\varphi^{k}=f_{k+1}+f_{k-1}-1+\frac{1}{1+\left(1 /\left(\varphi^{k}-1\right)\right)},
$$

from which the continued fraction of $\varphi^{k}$ easily follows.

\section{The General Difference Equation}

We now consider the more general difference equation

$$
G_{n}=m G_{n-1}+\ell G_{n-2}
$$

with the initial conditions $G_{0}=0, G_{1}=1$ (we need to choose initial conditions to explicitly write down a generalized Binet's formula). The results from Lemma 3 immediately generalize; we omit the proofs (which are available in [5]) which are straightforward algebra and just collect the results below. As before, we use both $G_{n}$ and $g_{k}$ for terms of the sequence to make the arguments below easier to visually parse.

Lemma 4. The authors' sequence $\left\{G_{n}\right\}$ satisfies the following properties.

(1) $G_{n}=m G_{n-1}+\ell G_{n-2}$ with $G_{0}=0, G_{1}=1$.

(2) $\varphi_{m, \ell}=\lim _{n \rightarrow \infty}\left(G_{n} / G_{n-1}\right)=\left(m \pm \sqrt{m^{2}+4 \ell}\right) / 2$.

(3) Generalized Binet's Formula:

$$
\begin{aligned}
G_{n}=\frac{1}{\sqrt{m^{2}+4 l}} & {\left[\left(\frac{m+\sqrt{m^{2}+4 \ell}}{2}\right)^{n}\right.} \\
& \left.-\left(\frac{m-\sqrt{m^{2}+4 l}}{2}\right)^{n}\right] .
\end{aligned}
$$

(4) $G_{n-2} G_{n}-\left(G_{n-1}\right)^{2}=(-1)^{n-1} \cdot \ell^{k-2}$.

(5) $G_{n}=g_{k+1} G_{n-k}+\lg _{k} G_{n-k-1}$.

We want to find closed form expressions for the continued fractions of the $\varphi_{m, \ell}^{k}$ for integral $k$. As the continued fraction of $\alpha$ is trivially related to that of $1 / \alpha$, it suffices to study $k>0$.
We argue, as in Section 2, from Lemma 4 (5) we have $G_{n}=$ $g_{k+1} G_{n-k}+\lg _{k} G_{n-k-1}$, and thus

$$
\begin{aligned}
\frac{G_{n}}{G_{n-k}} & =\frac{g_{k+1} G_{n-k}+\ell g_{k} G_{n-k-1}}{G_{n-k}} \\
& =g_{k+1}+\ell g_{k} \frac{G_{n-k-1}}{G_{n-k}} \\
& =g_{k+1}+\ell \frac{m g_{k-1} G_{n-k-1}+\ell g_{k-2} G_{n-k-1}}{G_{n-k}} \\
& =g_{k+1}+\ell\left(\left(m g_{k-1} G_{n-k-1}+\ell g_{k-1} G_{n-k-2}\right)\right. \\
& =g_{k+1}+\ell \frac{g_{k-1} G_{n-k}}{G_{n-k}}+\ell \frac{\ell g_{k-2} G_{n-k-1}-\ell g_{k-1} G_{n-k-2}}{G_{n-k}} \\
& =\left(g_{k+1}+\ell g_{k-1}\right)+\ell^{2} \frac{g_{k-2} G_{n-k-1}-g_{k-1} G_{n-k-2}}{G_{n-k}} .
\end{aligned}
$$

From Lemma 4 (5), we have $G_{n-k-1}=g_{k} G_{n-k-k}+$ $\ell_{g_{k-1}} G_{n-k-k-1}$. Also $G_{n-k-2}=g_{k-1} G_{n-k-k-1}+\ell_{g_{k-2}} G_{n-k-k-2}$. Thus, (26) reduces to

$$
\begin{aligned}
\frac{G_{n}}{G_{n-k}}= & \left(g_{k+1}+\ell g_{k-1}\right) \\
& +\ell^{2}\left(g_{k-2}\left(g_{k} G_{n-2 k}+\ell g_{k-1} G_{n-2 k-1}\right)\right. \\
& \left.\quad-g_{k-1}\left(g_{k-1} G_{n-2 k}+\ell g_{k-2} G_{n-2 k-1}\right)\right) \times\left(G_{n-k}\right)^{-1} \\
= & \left(g_{k+1}+\ell g_{k-1}\right)+\ell^{2} \frac{g_{k-2} g_{k} G_{n-2 k}-g_{k-1} g_{k-1} G_{n-2 k}}{G_{n-k}} \\
= & \left(g_{k+1}+\lg _{k-1}\right)+\ell^{2} \frac{G_{n-2 k}\left(g_{k-2} g_{k}-\left(g_{k-1}\right)^{2}\right)}{G_{n-k}} .
\end{aligned}
$$

From Lemma 4 (4) we know that

$$
g_{k-2} g_{k}-\left(g_{k-1}\right)^{2}=(-1)^{k-1} \ell^{k-2},
$$

and thus

$$
\frac{G_{n}}{G_{n-k}}=\left(g_{k+1}+\lg _{k-1}\right)+\frac{G_{n-2 k} \ell^{k}(-1)^{k-1}}{G_{n-k}} .
$$

We now prove our main result.

Proof of Theorem 2. Consider that we have the following cases.

Case $1(\ell=1)$. In this case, the difference equation takes the form $G_{n}=m G_{n-1}+G_{n-2}$. The functional form of (29) is identical to that of (18); the only difference is that we have $g$ 's and $G$ 's instead of $f$ 's and $F$ 's. We can thus quote our results from that case and obtain the desired result. 
Case $2(\ell=-1)$. When $\ell=-1$ the difference equation is of the form $G_{n}=m G_{n-1}-G_{n-2}$; we choose the initial conditions so that no term is ever negative. Taking $\ell=-1$ in (29) gives

$$
\begin{aligned}
\frac{G_{n}}{G_{n-k}} & =\left(g_{k+1}+g_{k-1}\right)+(-1)^{k-1}(-1)^{k} \frac{G_{n-2 k}}{G_{n-k}} \\
& =\left(g_{k+1}+g_{k-1}\right)-\frac{G_{n-2 k}}{G_{n-k}} .
\end{aligned}
$$

As this is of the same form as what we had in (20), we can argue as in Section 2. We have thus shown that all quadratic surds of the form $\varphi_{m,-1}=\left(m+\sqrt{m^{2}-4}\right) / 2$, and their powers, have continued fraction expansions of the form

$$
\varphi_{m,-1}^{k}=\left(g_{k+1}-g_{k-1}-1\right)+\frac{1}{1+\left(1 /\left(\varphi_{m,-1}^{k}-1\right)\right)} .
$$

This clearly determines a continued fraction with repeating block of length 2 of the following form:

$$
\varphi_{m,-1}^{k}=\left[g_{k+1}-g_{k-1}-1, \overline{1,\left(g_{k-1}-g_{k-1}-2\right)}\right] .
$$

This is a completing of the proof.

\section{Acknowledgment}

Steven J. Miller was partially supported by NSF grant DMS0970067. Daniel Fishman and Steven J. Miller would like to thank the students and faculty from the Fall 2002 and Spring 2003 undergraduate research lab at Princeton, where much of this work was done as part of Princeton University's VIGRE grant. This paper is dedicated to the memory of Alf van der Poorten.

\section{References}

[1] A. J. van der Poorten, "Fractions of the period of the continued fraction expansion of quadratic integers," Bulletin of the Australian Mathematical Society, vol. 44, no. 1, pp. 155-169, 1991.

[2] G. H. Hardy and E. Wright, An Introduction to the Theory of Numbers, Oxford Science Publications, Clarendon Press, Oxford, UK, 5th edition, 1995.

[3] S. J. Miller and R. Takloo-Bighash, An Invitation to Modern Number Theory, Princeton University Press, Princeton, NJ, USA, 2006.

[4] B. Gosper, "Continued Fraction Arithmetic (Unfinished)," http://www.tweedledum.com/rwg/cfup.htm.

[5] D. Fishman, "Closed Form Continued Fraction Expansions of Special Quadratic Irrationals, junior thesis (advisor S. J. Miller), Princeton University, 2002-2003," http://www.math. princeton.edu/mathlab/jr02fall/Closed/spring/dfJP2.pdf.

[6] E. Bombieri and A. J. van der Poorten, "Continued fractions of algebraic numbers," in Computational Algebra and Number Theory (Sydney, 1992), vol. 325 of Mathematics and Its Applications, pp. 137-152, Kluwer Academic, Dordrecht, The Netherlands, 1995.

[7] R. P. Brent, A. J. van der Poorten, and H. J. J. te Riele, "A comparative study of algorithms for computing continued fractions of algebraic numbers," in Algorithmic Number Theory (Talence, 1996), vol. 1122 of Lecture Notes in Comput. Sci., pp. 35-47, Springer, Berlin, Germany, 1996.
[8] E. B. Burger and A. J. van der Poorten, "On periods of elements from real quadratic number fields," in Constructive, Experimental, and Nonlinear Analysis (Limoges, 1999), vol. 27 of Conference Proceedings, Canadian Mathematical Society Series, pp. 35-43, American Mathematical Society, Providence, RI, USA, 2000.

[9] A. J. van der Poorten, "An introduction to continued fractions," in Diophantine Analysis (Kensington, 1985), vol. 109 of London Mathematical Society Lecture Note Series, pp. 99-138, Cambridge University Press, Cambridge, UK, 1986.

[10] A. J. van der Poorten, "Notes on continued fractions and recurrence sequences," in Number Theory and Cryptograph (Sydney, 1989), vol. 154 of London Mathematical Society Lecture Note Series, pp. 86-97, Cambridge University Press, Cambridge, UK, 1990.

[11] A. J. van der Poorten, "Continued fractions of formal power series," in Advances in Number Theory (Kingston, ON, 1991), pp. 453-466, Oxford Science Publications, Oxford University Press, New York, NY, USA, 1993.

[12] A. J. van der Poorten, "Continued fraction expansions of values of the exponential function and related fun with continued fractions," Nieuw Archief voor Wiskunde, vol. 14, no. 2, pp. 221-230, 1996.

[13] A. van der Poorten, Notes on Fermat's Last Theorem, Canadian Mathematical Society Series of Monographs and Advanced Texts, Wiley-Interscience, New York, NY, USA, 1996.

[14] A. J. van der Poorten and J. Shallit, "Folded continued fractions," Journal of Number Theory, vol. 40, no. 2, pp. 237-250, 1992.

[15] A. van der Poorten and J. Shallit, "A specialised continued fraction," Canadian Journal of Mathematics, vol. 45, no. 5, pp. 1067-1079, 1993.

[16] S. Louboutin, R. A. Mollin, and H. C. Williams, "Class numbers of real quadratic fields, continued fractions, reduced ideals, prime-producing quadratic polynomials and quadratic residue covers," Canadian Journal of Mathematics, vol. 44, no. 4, pp. 824-842, 1992.

[17] T. Koshy, Fibonacci and Lucas Numbers with Applications, Pure and Applied Mathematics, Wiley-Interscience, New York, NY, USA, 2001. 


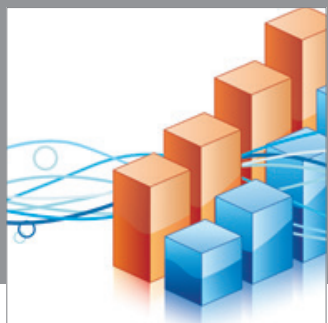

Advances in

Operations Research

mansans

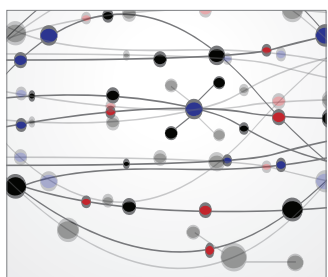

The Scientific World Journal
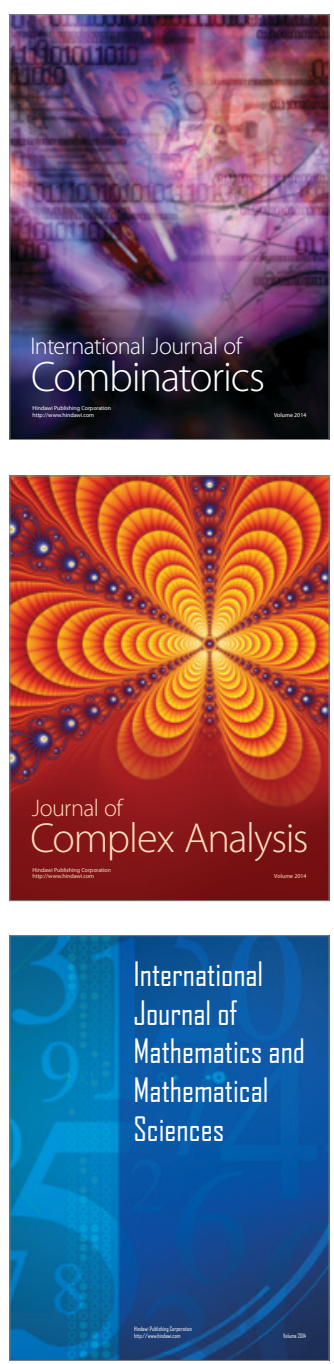
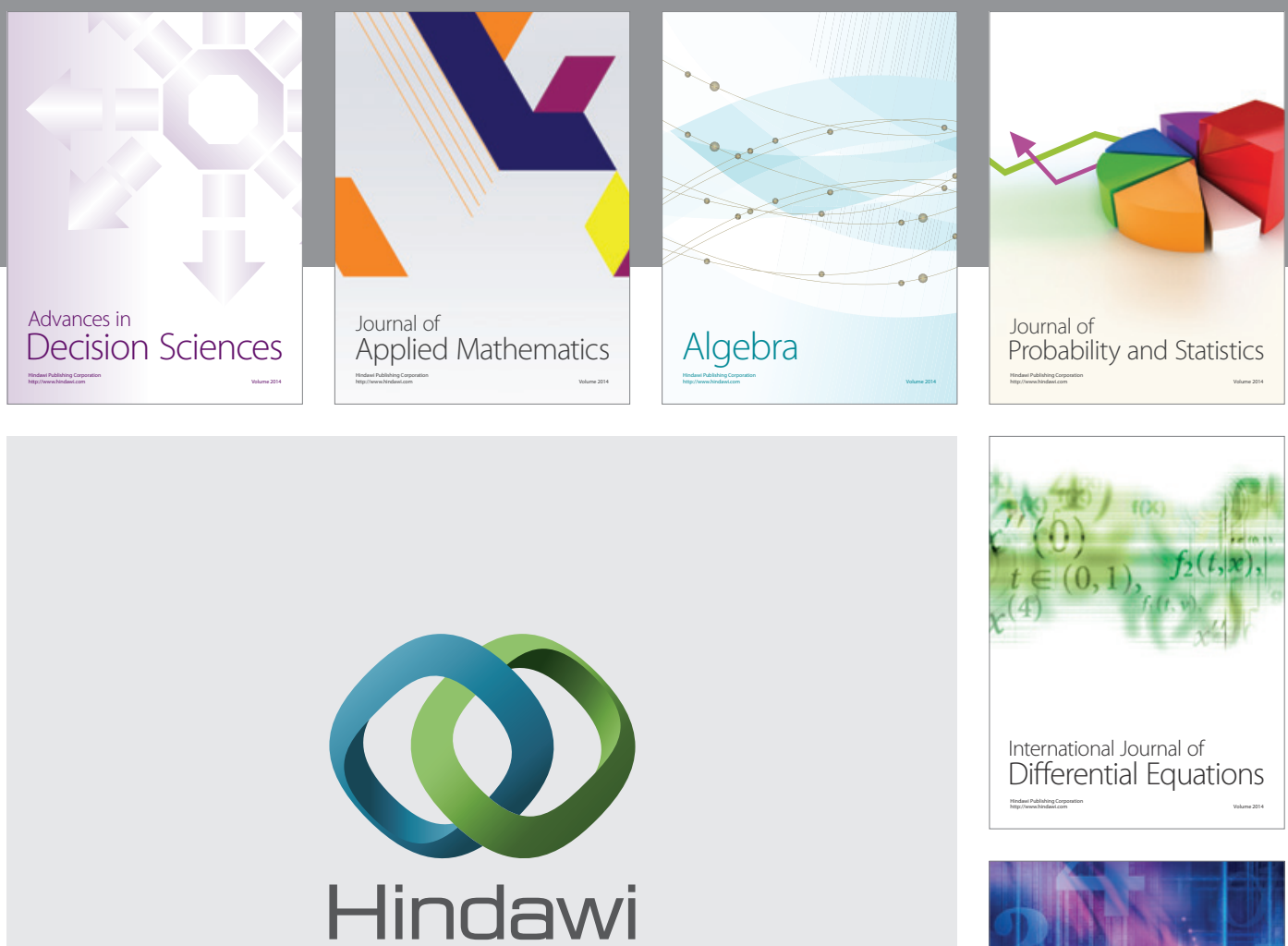

Submit your manuscripts at http://www.hindawi.com
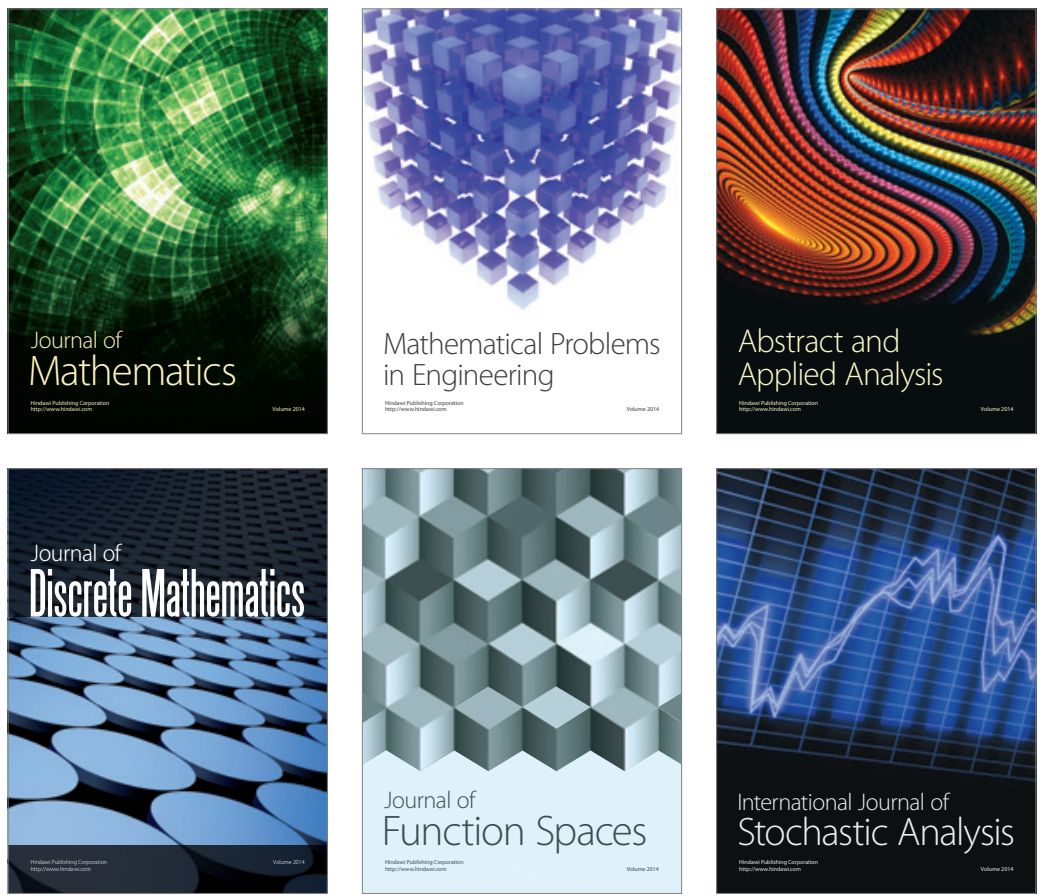

Journal of

Function Spaces

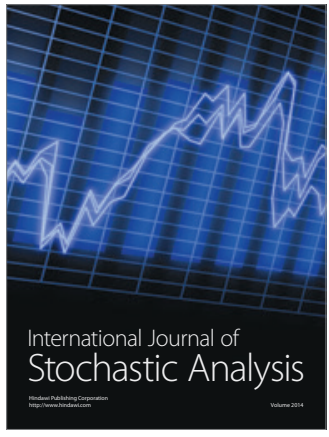

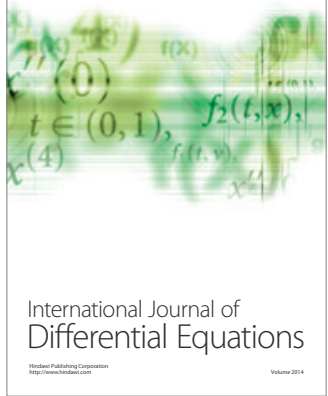
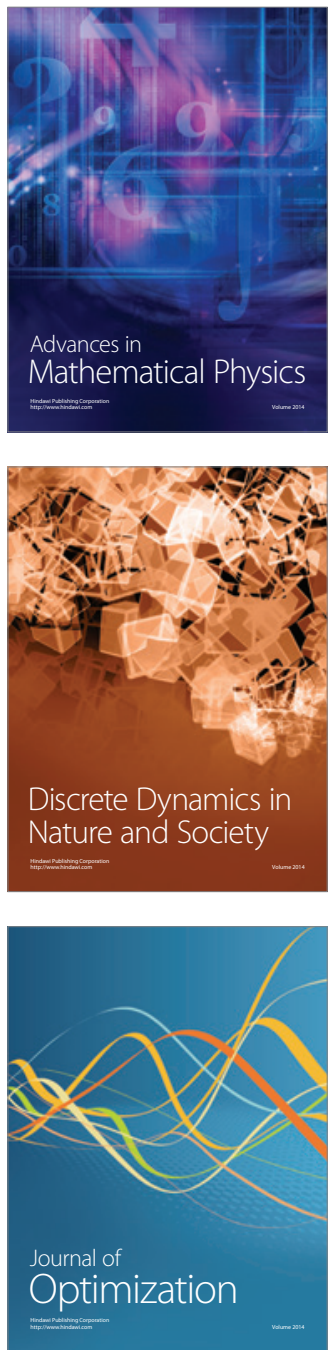